\title{
Establishment of Eichlinia gen.n. for the Western hemisphere Melittiini (Lepidoptera: Sesiidae), with a catalogue of the genus
}

\author{
Установление Eichlinia gen.n. в Melittiini (Lepidoptera: Sesiidae) \\ Западного полушария с каталогом рода
}

\author{
O.G. Gorbunov \\ О.Г. Горбунов
}

\begin{abstract}
A.N. Severtsov Institute of Ecology and Evolution, Russian Academy of Sciences, Leninsky prospekt 33, Moscow 119071, Russia. E-mail: gorbunov.oleg@mail.ru.

Институт проблем экологии и эволюции им. А.Н. Северцова РАН, Ленинский проспект 33, Москва 119071, Россия.
\end{abstract}

KEY WORDS: Melittia, Eichlinia, Nearctic Region, Neotripical Region, systematic, taxonomy, new combinations.

КЛЮЧЕВЫЕ СЛОВА: Melittia, Eichlinia, Неарктический регион, Неотропический регион, систематика, таксономия, новые комбинации.

ABSTRACT. A new genus from the tribe Melittiini (Lepidoptera, Sesiidae) is erected from the Western hemisphere: Eichlinia gen.n. The new genus differs from the all known genera of the tribe in the combination of the characters of both male and female genitalia. A key for determination of all genera of the tribe Melittiini is presented. All Nearctic and some Neotropical species of Melittiini are transferred from the genus Melittia to the new one. A preliminary catalogue species of the new genus, which contains updated taxonomic information including the references to the original descriptions, information on name-bearing types, mostly complete bibliographies, data on host plants and current known distribution is provided.

РЕЗЮМЕ. Установлен новый род Eichlinia gen.n. в трибе Melittiini (Lepidoptera, Sesiidae) Западного полушария. Новый род отличается от всех известных родов трибы сочетанием признаков мужских и женских гениталий. Представлен ключ для определения всех родов трибы Melittiini. Bсе неарктические и часть неотропических видов трибы Melittiini переносятся из рода Melittia в новый род. Предлагается предварительный каталог видов нового рода, который включает данные о первоначальных описаниях, информацию о номенклатурных типах, почти полную библиографию и современные знания о распространении.

\section{Introduction}

The genus Melittia Hübner, 1819 ["1816"] is the largest sesiid genus of the tribe Melittiini Le Cerf, 1917 [Arita, Gorbunov, 1996a, b; Gorbunov, Arita, 1996, 1997; Pühringer, Kallies, 2004, 2017; Gorbunov, 2014,
2015, 2017, 2020; Kallies, 2020]. It was originally erected by Hübner [1816-1826: 128] with the only species Melittia anthedoniformis Hübner, 1819 [“1816"], which was considered to be proposed as a replacement name for Sphinx bombiliformis Stoll, 1782 (= Sphinx bombyliformis Stoll, 1782) - a primary junior homonym of Sphinx bombyliformis Linnaeus, 1758 (Lepidoptera: Sphingidae) [Fletcher, Nye, 1982; Arita, Gorbunov, 1995; Gorbunov, 2017]. Further, M. anthedoniformis turned out to be the junior subjective synonym for Sesia chalciformis Fabricius, 1793 [Hampson, 1892]. Hence, Melittia anthedoniformis Hübner, 1819 [“1816”] (= Sphinx bombyliformis Stoll, 1782 [nec Sphinx bombyliformis Linnaeus, 1758]) = Sesia chalciformis Fabricius, 1793) is the type species and it was fixed by monotypy in accordance with Article 68.3 [ICZN, 1999]. The type locality of Melittia bombyliformis (Stoll, 1782) is " ... op de Kust van Coromandel ... (... sur la Côte de Coromandel ..." [Stoll, 1782: 242] or east coast of the Hindustan Peninsula south of the Krishna River Delta to Cape Komorin. The type locality of Sesia chalciformis Fabricius, 1793 was cited in the original description as "Habitat Tranquebariae ...", that is the town of Tharangambadi on the Coromandel Coast of India.

For a reason unclear to me, Hampson [1892: 202] indicates Melittia satyriniformis Hübner 1831 ["1825"] "from N. America" as the type species of the genus Melittia. His point of view was supported by Beutenmüller [1901: 231] and Bartel [1912: 379]. But already in his work on Sesiidae of the Oriental and Afrotropical regions, Hampson [1919: 84] corrected his mistake and rightly indicated the type species of the genus Melittia. Perhaps this fact for a long time prevented the elucida-

How to cite this article: Gorbunov O.G. 2020. Establishment of Eichlinia gen.n. for the Western hemisphere Melittiini (Lepidoptera, Sesiidae), with a catalog of the genus // Russian Entomol. J. Vol.29. No.3. P.276-284. doi: 10.15298/rusentj.29.3.06 
tion of the actual generic position of the Melittiini species of North America. Only in the mid-90s of the last century it was indicated that they all have clear differences in the structure of the male genitalia from Melittia from the Old World and all of them should be separated from it [Arita, Gorbunov, 1996b; Gorbunov, Arita, 1997].

In 1916-17 Le Cerf described three new genera of Melittiini from South America, namely Premelittia Le Cerf, 1916 (type species: Premelittia rufescens Le Cerf, 1916), Neosphecia Le Cerf, 1916 (type species: Neosphecia combusta Le Cerf, 1916), and Melittina Le Cerf, 1917 (type species Melittina nigra Le Cerf, 1917) [Le Cerf, 1916; 1917].

In their publication regarding a classification of Sesiidae of America north of Mexico, Duckworth and Eichlin [1977] absolutely without any argument synonymized these three taxa with the genus Melittia Hübner, 1819 ["1816"]. In 2017 I confirmed that the representatives of the Neotropical taxa of Melittiini "do not belong to Melittia and must be placed elsewhere" [Gorbunov, 2017: 128]. More recently, a review of the Neotropical genera of Melittiini described by Le Cerf was published, which restored all of them from synonyms of the genus Melittia [Moreira et al., 2019]. And now it is completely clear to me that all Melittiini species of North America as well as some of those from the Neotropical region belong to another genus that has not yet been described. Below I describe it as Eichlinia gen.n. In addition, I provide a preliminary catalogue of the genus Eichlinia gen.n., which contains updated taxonomic information including the references to the original descriptions, information on name-bearing types, mostly complete bibliographies, data on host plants and current known distribution. All synonymic names in the text are given in quotation marks, in the same way as they are presented in the original descriptions. The names of the host plants were verified with the Plant List [2010].

The images of moths were taken with a Sony ${ }^{\circledR} \alpha 450$ DSLR camera equipped with a Minolta ${ }^{\circledR} 50 \mathrm{f} / 2.8$ Macro lens. The genitalia were photographed using a Keyence ${ }^{\circledR}$ BZ-9000 Biorevo Fluorescence Microscope. The processing of all illustrations was finalized with Adobe ${ }^{\circledR}$ Photoshop ${ }^{\circledR}$ CC 2020 software.

All images of dry specimens are labeled with a number consisting of letters and digits: name of the family, two consecutive digits separated by n-dash and a year following m-dash (e.g. SESIIDAE Pictures №№ 0473-0474-2019). These letters and digit codes correspond to the numbering system of the figured specimens in the author's archive.

Genitalia preparations are stored in microtubes with glycerol and pinned under the specimens. A label with the number of the genital preparation as a label (e.g. Genitalia preparation № OG-059-2018) is attached under each specimen and is listed in the author's archive.

The material studied or mentioned herein is deposited in the following collections abbreviated in the text as follows:
AMNH - the American Museum of Natural History, New York, USA;

BMNH - the Natural History Museum, London, UK; CIML — Instituto Miguel Lollo, Tucuman, Argentina; COGM - A.N. Severtsov Institute of Ecology and Evolution of Russian Academy of Sciences, Moscow, Russia;

FMNH - Field Museum of Natural History, Chicago, Illinois, USA;

MACN - Museo Argentino de Ciencias Naturales Bernardino Rivadavia, Buenos Aires, Argentina;

MNHP - Muséum national d'Histoire naturelle, Paris, France;

MCZC - Museum of Comparative Zoology, Cambridge, Massachusetts, USA;

NMNH - National Museum of Natural History, Smithsonian Institution, Washington DC, USA;

ZNHB - Museum für Naturkunde, Zentralinstitut der Humboldt-Universität zu Berlin, Germany.

\section{Taxonomic account}

\section{Eichlinia O. Gorbunov, gen.n.} Figs 1-4, 9-15.

TYPE SPECIES: Aegeria cucurbitae Harris, 1828 (Figs 1-4). Melittia auct., nec Melittia Hübner, 1819 ["1816"]

DESCRIPTION. Colourful, rarely medium-sized, but mostly large-sized clearwing moths with alar expanse 24-62 $\mathrm{mm}$. Superficially resembling genus Melittia (Figs 5-8).

Head with antenna slightly clavate with a poorly defined hook distally, ciliate and sometimes unipectinate in male; frons smooth-scaled with a projection of flat scales beneath antenna externally forming a characteristic shelf; labial palpus long, turhed-up, with a few hair-like scales ventrally; proboscis well-developed, long, functional; vertex covered with short hair-like scales. Thorax smooth-scaled, both metepimeron and metameron with long hair-like scales posteriorly. Hind leg with tibia and two basal tarsomeres tufted with long hair-like scales. Abdomen smooth-scaled, anal tuft undeveloped. Forewing completely opaque or posterior transparent area present but narrow and very short; veins $R_{1}-R_{3}$ parallel, $R_{4}$ and $R_{5}$ stalked for about half of their length; distance between bases of veins $R_{4+5}, M_{1}, M_{2}$ and $M_{3}$ nearly equal (Fig. 9). Hindwing transparent or opaque; vein $\mathrm{M}_{3}$ arises from vein $\mathrm{CuA}_{1}$ distinctly basal of cross-vein (Fig. 9).

Male genitalia. Tegumen-uncus complex broad; uncus broadened and bilobed distally with a large semi-circular plate of strong, short, pointed setae internally on each side; gnathos undeveloped (Fig. 10); valva (Fig. 11) elongateoval, sometimes distinctly pointed ventro-apically, with a small group of short dense setae dorso-apically; saccular region with 1-3 projecting ridges devoid of setae; saccus broad and long, slightly club-shaped and rounded basally (Fig. 12); aedeagus (Fig. 13) narrow, somewhat longer than valva; vesica (Fig. 14) with numerous, small, pointed cornuti.

Female genitalia. $8^{\text {th }}$ tergite narrow; lamella postvaginalis $\mathrm{V}$-shaped, well scleritized, lamella antevaginalis undeveloped; ostium bursae at level of $8^{\text {th }}$ tergite, membranous, slightly funnel-shaped membranous; antrum membranous, thin and long, about as long as ostium bursae; corpus bursae ovoid without or with a small signum of minute spinules (Fig. 15). 
DIFFERENTIAL DIAGNOSIS. Superficially, species of this new genus resemble certain species of genus Melittia with completely opaque forewings (M. astarte (Westwood,

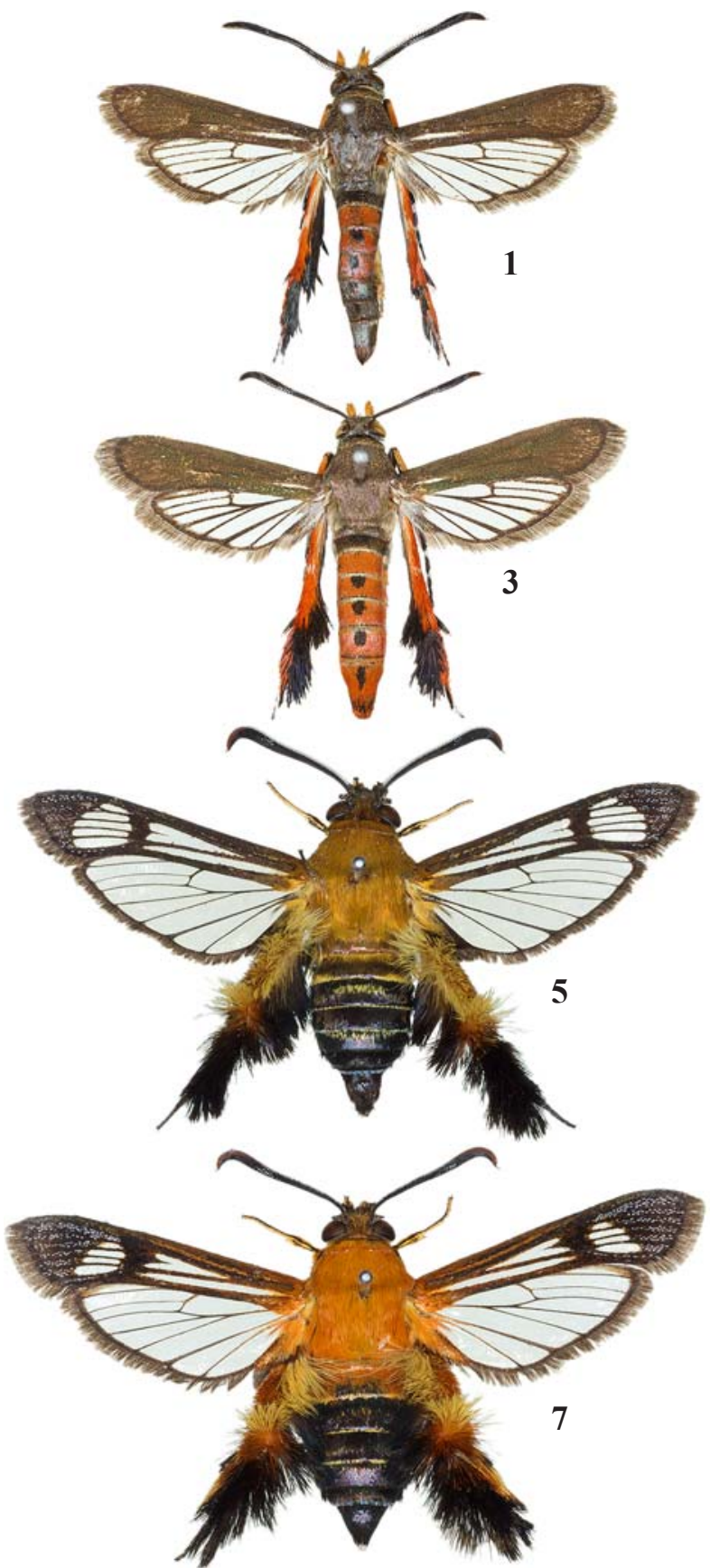

1848) species-group and M. aureosquamata (Wallengren, 1863) species-group), and Afromelittia caerulea Bartsch, 2016. However, by the structure of the male genitalia Eichlin-

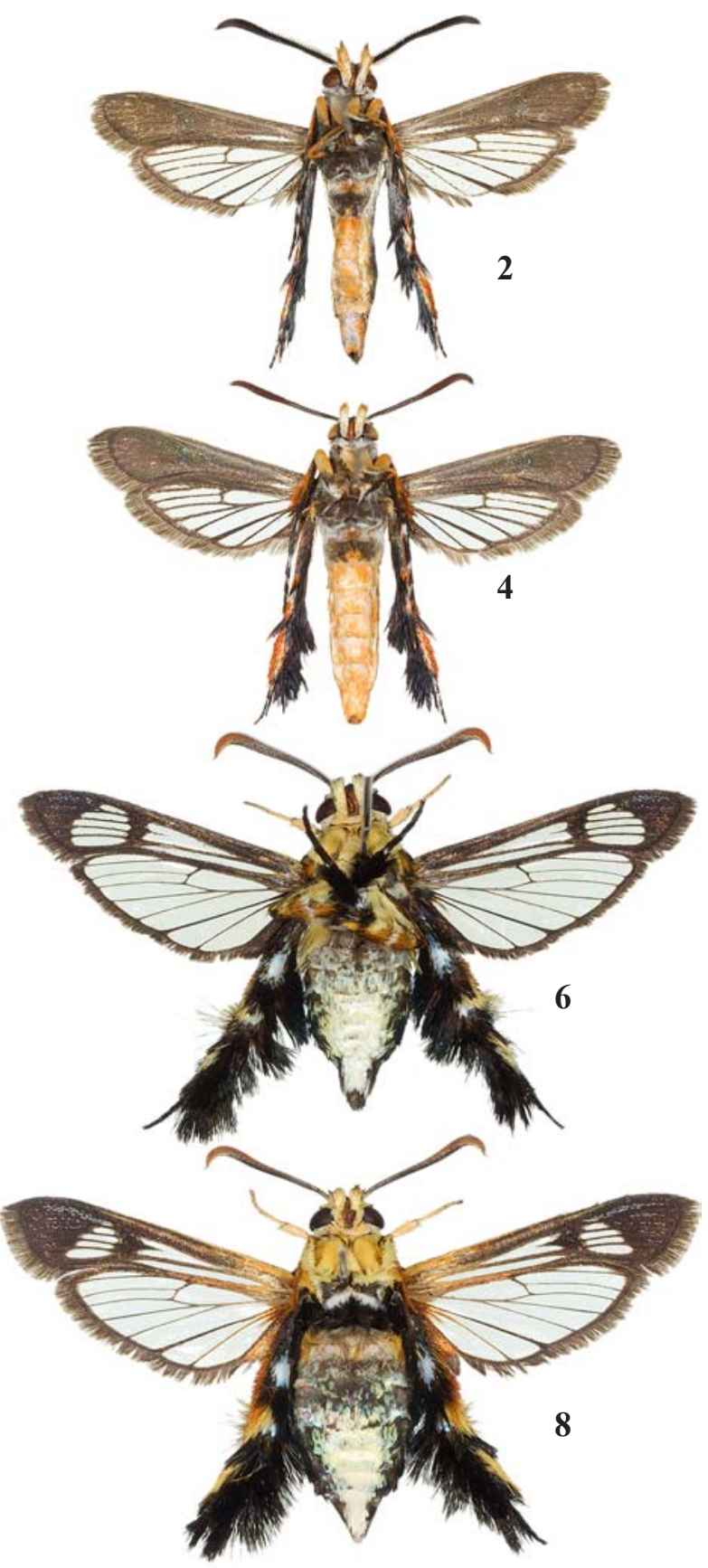

Figs 1-8. Melittiini spp: 1-4 - Eichlinia cucurbitae (Harris, 1828), comb.n.; 1-2 - male, USA, Montana, Columbus, 21.VI01.VII.1994, native collector. Sesiidae picture № 0473-0474-2019. Alar expanse $25.2 \mathrm{~mm}$ (COGM); 3-4 - female, USA, Montana, Columbus, 21.VI-01.VII.1994, native collector. Sesiidae picture № 0469-0470-2019. Alar expanse $26.0 \mathrm{~mm}$ (COGM); 5-8 - Mellitia sangaica nipponica Arita et Yata, 1987; 5-6 - male, Japan, Honshu, Aichi-ken, Toyota-shi, 07.I.2002, ex l., O. Gorbunov \& B. Tanaka leg. Sesiidae picture № 0061-0062-2014. Alar expanse 41.5 mm (COGM); 7-8 - female, Japan, Honshu, Aichi-ken, Kasugai-shi, Takagi, 18.I.2002, ex l., O. Gorbunov \& Y. Arita leg. Sesiidae picture № 0053-0054-2014. Alar expanse $42.0 \mathrm{~mm}$ (COGM).

Рис. 1-8. Melittiini spp: 1-4 - Eichlinia cucurbitae (Harris, 1828), comb.n.; 1-2 - самец, США, Монтана, Коламбас, 21.VI01.VII.1994, местный коллектор. Sesiidae снимки № 0473-0474-2019. Размах крыльев 25,2 мм (COGM); 3-4 - самка, США, Монтана, Коламбас, 21.VI-01.VII.1994, местный коллектор. Sesiidae снимки № 0469-0470-2019. Размах крыльев 26,0 мм (COGM); 5-8 - Mellitia sangaica nipponica Arita et Yata, 1987; 5-6 - самец, Япония, Хоншю, префектура Айчи, Тоёта, 07.I.2002, ex l., О.Горбунов, Б. Танака leg. Sesiidae снимки № 0061-0062-2014. Размах крыльев 41,5 мм (COGM); 7-8 - самка, Япония, Хоншю, префектура Айчи, окрестности Касугайя, Такаги, 18.I.2002, ex l., О.Горбунов, Ю. Арита leg. Sesiidae снимки № 0053-0054-2014. Размах крыльев 42,0 мм (COGM). 
ia gen.n. seems to be the closest to Afromelittia Gorbunov et Arita, 1997 (type species: Melittia occidentalis Le Cerf, 1917), but they can be distininguished by both male and female genitalia (somewhat different shape of the tegumenuncus complex and valva in the male, position of the ostium bursae, shape of the lamella postvaginalis, structure of the antrum and the shale of corpus bursae. Compare Figs 10-15 with figs 43 and 44 [in Gorbunov, Arita, 1997] or with figs 13a-g [in Eichlin, Duckworth, 1988]. From the genus Melittia, the species of the genus Eichlinia gen.n. clearly differ also by the structure of both male and female genitalia [compare Figs 10-15 and figs 13a-g in Eichlin, Duckworth, 1988 with numerous figs in Arita, Gorbunov, 1995, 1996a, b; Gorbunov, Arita, 1996, 1997; Špatenka et al., 1999; Gorbunov, 2014, 2015, 2017, 2020; Bartsch, 2016].

All genera of the tribe Melittiini can be determined by the following key compiled by the combinations of external features, morphology of both male and female genitalia and zoogeographical affiliation. I exclude from this key a completely unstudied monotypical genus Desmopoda Felder et Felder, 1874 (type species: Desmopoda bombiformis Felder et Felder, 1874), which is still know by a single holotype $(+)$ originated from the island of Ambon, Maluku, Indonesia. Highly likely, Desmopoda is a junior synonym of Melittia [Arita, Gorbunov, 1995; Gorbunov, 2020].

1 Proboscis undeveloped... .. 2

- Proboscis well-developed

2 Vertex covered with hair-like scales; forewing with vein $\mathrm{R}_{4}$ and $\mathrm{R}_{5}$ separate basally; Neotropical..... Neosphecia Le Cerf, 1916

- Vertex smoothly scaled; forewing with veins $R_{4}$ and $R_{5}$ short stalked; Neotropical.... Premelittia Le Cerf, 1916
3 Head broad, distance between external margins of eyes larger than maximum width of protorax; anal lobe of hindwing transparent; Oriental

Cephalomelittia Gorbunov et Arita, 1995

- Head normal, distance between external margins of eyes less than maximum width of protorax; anal lobe of the hindwing opaque or undeveloped .............................. 4

4 Forewing with veins $\mathrm{R}_{3}$ and $\mathrm{R}_{4+5}$ short stalked ........... 5

- Forewing with veins $\mathrm{R}_{3}$ and $\mathrm{R}_{4+5}$ separate basally ...... 6

5 Forewing with transparent areas very small or completely undeveloped; male genitalia with uncus bilobed, broadened and rounded distally, with a large plate of short but strong setae on each side internally, valva rounded or pointed ventro-apically, with straight ventral margin [see figs 44a, 44d in Gorbunov, Arita, 1997 or figs 11-13 in Bartsch, 2016]; female genitalia with lamella postvaginalis well-developed, antrum slightly funnel-shaped, well-sclerotized medially [see fig. 43 in Gorbunov, Arita, 1997]; Afrotropical ........ Afromelittia Gorbunov et Arita, 1997

- Forewing with transparent areas we-developed; male genitalia with uncus bilobed and pointed apically, with a few teeth at tip internally, valva narrowed medially, with a small rounded appendix distally [see figs 30, 40, 41 in Gorbunov, Arita, 1997]; female genitalia with lamella postvaginalis undeveloped, antrum tube-shaped, wellsclerotized posteriorly [see fig. 42 in Gorbunov, Arita, 1997]; Afrotropical .......... Agriomelissa Meyrick, 1931

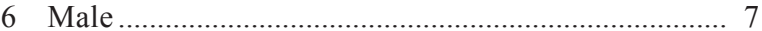

— Female ....................................................................... 9

7 Genitalia with uncus distally without a plate of strong, short, pointed setae internally on each side, valva narrowly elongated dorso-apically, sometimes with a finger-
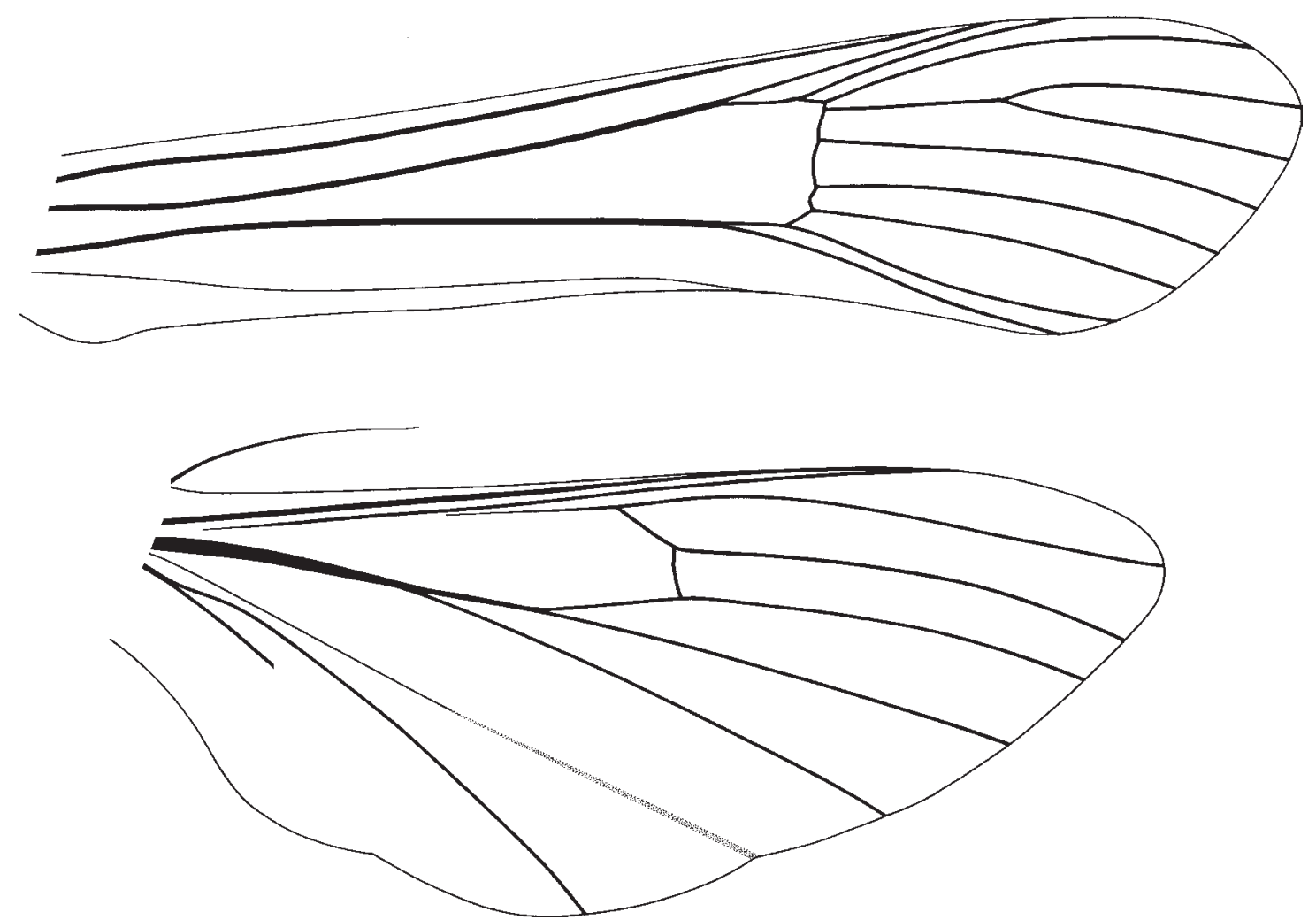

Fig. 9. Wing venation of Eichlinia cucurbitae (Harris, 1828), comb.n.

Рис. 9. Жилкование крыльев Eichlinia cucurbitae (Harris, 1828), comb.n. 
shaped appendix medio-ventrally, covered with sparse setae at dorsal margin [see figs 7a, 7b in Gorbunov, Arita, 1995 or fig. 10 in Kallies et al., 2016]; East Palaearctic, Oriental . Macroscelesia Hampson, 1919

- Genitalia with uncus distally with a plate of strong, short, pointed setae internally, valva, oval, elongate-oval or rectangular with 1-3 groups of dense setae distally

8 Antenna with well-defined hook distally; uncus narrowed distally, gnathos present, valva oval, elongate-oval or rectangular with 3 groups of dense setae; Afrotropical, East Palaearctic, Oriental, Australasian Melittia Hübner, 1819 [“1816”]

- Antenna with poorly defined hook distally; uncus broadened distally, gnathos undeveloped, valva oval or elongate-oval with a group of dense setae; Nearctical, Neotropical Eichlinia O. Gorbunov, gen.n.
9 Legs with hind tibia and tarsus with slightly elongated scales, not forming a well defined hindleg tuft; hindwing with anal lobe undeveloped; Neotropical

Melittina Le Cerf, 1917

- Legs with hind tibia and tarsus with elongated scales, forming a well defined hindleg tuft; hindwing with anal lobe present

10

10 Ostium bursae at level of $8^{\text {th }}$ tergite (Fig. 15); Nearctical, Neotropical ................... Eichlinia O. Gorbunov, gen.n.

- Ostium bursae at $7^{\text {th }}$ sternite ... 11

11 Ostium bursae opening at posterior half of $7^{\text {th }}$ sternite, funnel-shaped, slightly sclerotized; corpus bursae ovoid or pear-shaped with a more or less developed signum, forming from rows of minute spinules [see figs 312-315 in Špatenka et al., 1999]; Afrotropical, East Palaearctic, Oriental, Australasia

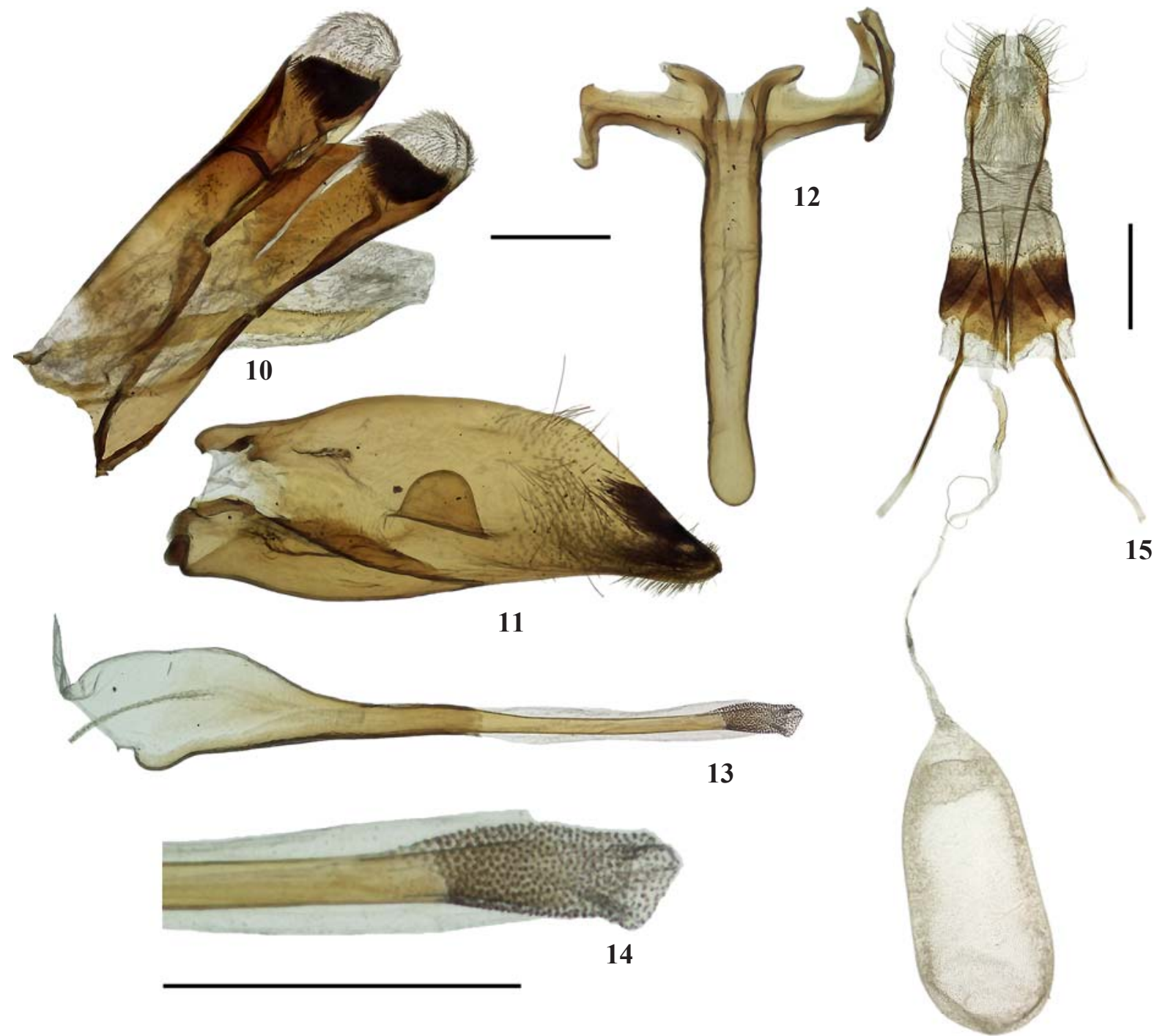

Figs 10-15. Genitalia of Eichlinia cucurbitae (Harris, 1828), comb.n.: 10-14 - male, USA, Montana, Columbus, 21.VI-1.VII.1994, local collector (COGM).Genital preparation № OG-059-2018; 10 - tegumen-uncus complex; 11 — valva; 12 - saccus; 13 - aedeagus; 14 - vesica; 15 - female, USA, Montana, Columbus, 21.VI-1.VII.1994, local collector (COGM). Genital preparation № OG-060-2018; Scale bar $0.5 \mathrm{~mm}, 1.0 \mathrm{~mm}$ for 15 .

Рис. 10-15. Гениталии Eichlinia cucurbitae (Harris, 1828), comb.n.: 10-14 - самец, США, Монтана, Коламбас, 21.VI-1.VII.1994, местный сборщик (COGM). Препарат гениталий № OG-059-2018; 10 — тегумен-ункус комплекс; 11 — вальва; 12 — саккус; 13 — эдеагус; 14 - везика; 15 - самка, США, Монтана, Коламбас, 21.VI-1.VII.1994, местный сборщик (COGM). Препарат гениталий № OG-060-2018; масштаб: 0,5 мм, 1,0 мм для 15 . 
- Ostium bursae opening at anterior margin of $7^{\text {th }}$ sternite, cup-shaped, well-sclerotized; corpus bursae globose without signa [see figs 11, 12 in Kallies et al., 2016]; East Palaearctic, Oriental ...... Macroscelesia Hampson, 1919

LIFE HISTORY. Due to the fact that some species of Eichlinia gen.n. are serious pests of pumpkins (Cucurbitaceae), the features of their biology are well known and described in detail. In accordance of the Western hemisphere investigations [Engelhardt, 1946; Eichlin, 1975; Eichlin, Duckworth, 1988; Canhilal et al., 2006] the larval host plants are species of the family Cucurbitaceae, including cultivars. Larvae are stem borers. They live in the lower part of vine where sometimes form a gall-like broadenings. The fully grown larva burrows into the topsoil, where it makes a cocoon. The larva overwinters in this earthen cocoon and pupates in early summer of the following year. There are one or two generations, depending on the natural conditions.

COMPOSITION. I am currently including 11 species in this new genus. The most important information about all these types is presented in the catalog below.

RANGE. Nearctic and Neotropical regions from NE Canada (Ontario) in the north to Argentina (Buenos Aires) in the south.

ETYMOLOGY. The new genus is dedicated to the late Dr. Thomas D. Eichlin (21.09.1938-19.09.2013), a major authority on the family Sesiidae of the Western Hemisphere. The gender is feminine.

\section{Preliminary catalogue of the genus Eichlinia O. Gorbunov, gen.n.}

\section{Eichlinia calabaza (Duckworth et Eichlin, 1973), comb.n.}

"Melittia calabaza n. sp." — Duckworth, Eichlin, 1973b: 151, figs 3a, 6; map. 1. Type locality: "Mexico: Mex., Teotihuacan, ...". Holotype $\sigma^{7}$ (USNM).

Mellitia calabaza - Duckworth, Eichlin, 1977: 52; Duckworth, Eichlin, 1978a: 7; Duckworth, Eichlin, 1978b: 5, 21, figs 4 , 20, pls 2, 8; Solomon, Dix, 1979: 16; Heppner, Duckworth, 1981: 26; Sondak, 1981, figs 1-7; Becker, Eichlin, 1984: 13, 14; Friedlander, 1986: 282, 283; Eichlin, Duckworth, 1988: 52, 54, text-figs 13a, 13c, 13g, pl. 2, fig. 2; Eichlin, 1995: 48; Pühringer, Kallies, 2004: 17; Pohl et al., 2016: 206; Moreira et al., 2019: 42, 43; San Martín-Romero et al., 2019.

HOST PLANT. C. argyrosperma K. Koch, Cucurbita maxima Duchesne, $C$. moschata Duchesne, $C$. pepo L. $(\mathrm{Cu}-$ curbitaceae).

DISTRIBUTION. USA: Arizona, New Mexico, Texas; Mexico: Zacatecas, Hidalgo, Mexico, Morelos, Veracruz.

Eichlinia cucurbitae (Harris, 1828), comb.n.

“Aegeria Cucurbitae." - Harris, 1828: 33. Type locality: not stated [USA]. Holotype + (MCZC).

= "Melittia Satyriniformis." — Hübner, 1831 [“1825"]: 17, figs 453, 454. Type locality: “... das Vaterland ... Georgien ist." [= USA: Georgia]. Type material: lost.

= "Trochilium ceto. Westw." — Westwood, 1848: 62, pl. 30, fig. 6. Type locality: "North America". Holotype + (BMNH).

= "Melittia Amoena. n. sp." — Edwards, 1882: 53. Type locality: "Douglas Co., Kansas, 900 feet, ..." [= USA: Kansas, Douglas County]. Type material: lost.

Aegeria cucurbitae — Engelhardt, 1946: 182; Duckworth, Eichlin, 1973a: 9.

Melittia amoena — Beutenmüller, 1896: 113; Duckworth, Eichlin, 1973a: 3.

Melittia cucurbitae — Beutenmüller, 1896: 113; Dalla Torre Strand, 1925: 140; Zukowsky, 1936: 1248, pl. 179, row b; McDunnough, 1939: 86; Engelhardt, 1946: 182, 183, 216, pl. 3, fig. 23; pl. 12, fig. 54, 54a; pl. 16, fig. 84; Howe, 1950: 480, 483; Howe, Rhodes,
1973: 266; Heppner, Duckworth, 1981: 26; Eichlin, Duckworth, 1988: 52, text-figs 12a, 12b, 12c, 12d, 13b, pl. 2, fig. 4; Klun et al., 1990: 64; Brown, Mizel, 1993: 6, 8, 16, pl. 2, figs 12a-b; Becker, Eichlin, 1984: 13, 14; Friedlander, 1986: 283; Eichlin, 1995: 48; Pühringer, Kallies, 2004: 17; Jackson et al., 2005: 27; Canhilal et al., 2006: 1; Capinera, 2008: 3533; Krinski, 2015: 1; Pohl et al., 2016: 206; Middleton, 2018: 1; Moreira et al., 2019: 42, 43.

Melittia satyriniformis — Druce, 1883: 32; Druce, 1896: 324; Beutenmüller, 1899: 149; Beutenmüller, 1901: 228, 229, 232, fig. 8, pl. 29, fig. 1; Dyar, 1902: 364; Walsingham, 1913: 192; Dalla Torre, Strand, 1925: 148; Zukowsky, 1936: 1249, pl. 179, row b; McDunnough, 1939: 86; Duckworth, Eichlin, 1977: 52; Solomon, Dix, 1979: 16; Becker, Eichlin, 1984: 13, 14.

Melittia ceto - Walker, 1856: 66; Druce, 1883: 32; Engelhardt, 1946: 182

Trochilium ceto — Engelhardt, 1946: 182; Duckworth, Eichlin, 1973a: 7.

HOST PLANT. Cucurbita andreana Naudin, C. argyrosperma K. Koch, C. ecuadorensis Cutler et Whitaker, C. ficifolia Bouché, C. maxima Dochesne, C. moschata Dochesne, $C$. okeechobeensis (Small) L.H. Bailey, C. pepo L., Echinocystis lobata (Michx.) Torr. et A. Gray (Cucurbitaceae).

DISTRIBUTION. Canada: Ontario; USA: Montana, Minnesota, Wisconsin, Michigan, New York, Vermont, Maine, South Dakota, Nebraska, Iowa, Illinois, Indiana, Ohio, Pennsylvania, New Jersey, Connecticut, Kansas, Missouri, Kentucky, West Virginia, Virginia, Oklahoma, Arkansas, Tennessee, North Carolina, Texas, Louisiana, Mississippi, Alabama, Georgia, South Carolina, Florida; Mexico: Coahuila, Durango, Jalisco, Mexico, Veracruz, Guerrero, Yucatan; Guatemala: Baja Verapaz; Panama: Chiriqui; Colombia; Venezuela; Peru; Brazil: Amazonas, Pará, São Paulo; Argentina: Buenos Aires.

Eichlinia eichlini (Friedlander, 1986), comb.n.

"Melittia eichlini new species Friedlander" — Friedlander, 1986: 278, figs 1, 2, 4, 7, 8, 11-16. Type locality: “...Jalisco, $5 \mathrm{~km}$ w. Atenquiqui, ..." [= Mexico: Jalisco, Atenquiqui]. Holotype $\sigma^{7}$ (NMNH).

Melittia eichlini — Eichlin, 1995: 48; Pühringer, Kallies, 2004: 15.

HOST PLANT. Cucurbita argyrosperma K. Koch (Friedlander [1986: 281, 283] cited as C. sororia Bailey) (Cucurbitaceae).

DISTRIBUTION. Mexico: Jalisco, Colima, Michoacan.

Eichlinia faulkneri (Eichlin, 1992), comb.n.

"Melittia faulkneri, new sp." — Eichlin, 1992: 141, figs 11, 29. Type locality: "Mexico.- Baja California: 49 mi. S Cataviña, ..." [= Mexico: Baja California, Ensenada, $78.9 \mathrm{~km}$ S Cataviña]. Holotype ○ (USNM).

Melittia faulkneri — Eichlin, 1995: 48; Brown, 2004: 102; Pühringer, Kallies, 2004: 15.

HOST PLANT. Cucurbita palmata S. Watson (Cucurbitaceae).

DISTRIBUTION. Mexico: Baja California.

Eichlinia gilberti (Eichlin, 1992), comb.n.

= "Melittia gilberti, new sp." — Eichlin, 1992: 140, figs 9, 10, 25. Type locality: "Mexico. - Baja California: 49 mi. S Cataviña, .." [= Mexico: Baja California, Ensenada, $78.9 \mathrm{~km} \mathrm{~S}$ Cataviña]. Holotype $\sigma^{7}$ (USNM).

Melittia gilberti — Eichlin, 1995: 48; Brown, 2004: 102; Pühringer, Kallies, 2004: 15.

HOST PLANT. Cucurbita palmata S. Watson (Cucurbitaceae).

DISTRIBUTION. Mexico: Baja California.

Eichlinia gloriosa (Hy Edwards, 1880), comb.n.

"Melittia Gloriosa, n. sp." — Edwards, 1880: 71. Type locality: “... in San Leonardo, Cal., ..." [= USA: California, Alamedo County, San Leonardo]. Holotype + (AMNH). 
$=$ "Melittia superba n. sp." — Barnes, Lindsey, 1922: 122. Type locality: “... Seward Co., Kansas.” [= USA: Kansas, Seward County]. Holotype $\sigma^{7}$ (USNM). It is a junior primary homonym of Melittia superba Rothschild, 1909.

="Melittia lindseyi, new name." — Barnes, Benjamin, 1925: 14. Replacement name for Melittia superba Barnes et Lindsey, 1922.

= "[Melittia $]$ Barnesi Dalla Torre nom. nov." - Dalla Torre in Dalla Torre, Strand, 1925: 138. Replacement name for Melittia superba Barnes et Lindsey, 1922.

Melittia barnesi - Duckworth, Eichlin, 1973a: 5.

Mellitia gloriosa — Beutenmüller, 1892: 171; Beutenmüller, 1896: 113; Beutenmüller, 1899: 149, 150; Beutenmüller, 1901: 235, pl. 29, fig. 3; Dyar, 1902: 364; Snow, 1905: 160; Engelhardt 1924: 125; Dalla Torre, Strand, 1925: 144; Thompson, 1929: 121; Zukowsky, 1936: 1249, pl. 179, row c; McDunnough, 1939: 87; Engelhardt, 1946: 182, 188, pl. 31, figs 178, 179; Duckworth, Eichlin, 1973a: 13; Duckworth, Eichlin, 1977: 52; Duckworth, Eichlin, 1978b: 5, 23, figs 5-7, 21, pl. 2; Solomon, Dix, 1979: 16 Heppner, Duckworth, 1981: 26; Friedlander, 1986: 282; Eichlin, Duckworth, 1988: 52, 57, text-fig. 13d, pl. 2, figs 1, 3, 5; Eichlin, 1995: 48; Brown, 2004: 102; Powell, 2005: 367; Pühringer, Kallies, 2004: 17; Taft, Schaper, 2014: 58, 60; Pohl et al., 2016: 206; Moreira et al., 2019: 42, 43.

Melittia lindseyi — Zukowsky, 1936: 1249; McDunnough, 1939: 87; Stallings, Turner, 1944: 30, 31; Duckworth, Eichlin, 1973a: 15, 28.

Melittia superba — Duckworth, Eichlin, 1973a: 5, 15, 28.

HOST PLANT. Cucurbita foetidissima Kunth, C. palmata S. Watson, Marah fabaceus (Naudin) Greene (Cucurbitaceae).

DISTRIBUTION. USA: Oregon, California, Kansas, Arizona, New Mexico, Texas, Colorado, Oklahoma. Mexico: Sonora, Baja California.

Eichlinia grandis (Strecker, 1881), comb.n.

"Trochilium Grande, n. sp." - Strecker 1881: 156. Type locality: "Hab. Texas." [= USA: Texas]. Holotype + (FMNH).

= "Melittia Beckeri, sp. n." — Druce, 1892: 276. Type locality: "Hab. Mexico, near Durango city ..." [= Mexico: Durango, Durango]. Holotype $\sigma^{7}$ (BMNH).

= "Melittia grandis hermosa, new variety" - Engelhardt, 1946: 186, pl. 31, fig. 177. Type locality: "Arizona." [= USA Arizana]. Holotype + (NMNH).

Melittia beckeri - Druce, 1896: 325; Druce, 1897: pl. 69, fig. 18; Duckworth, Eichlin 1978a: 5.

Mellitia grandis — Beutenmüller, 1896: 113; Beutenmüller, 1899: 149, 151; Beutenmüller, 1901: 235, pl. 29, fig. 4; Dyar, 1902 364; Walsingham, 1913: 193; Dalla Torre, Strand, 1925: 144; Zukowsky, 1936: 1249, pl. 179, row c; McDunnough, 1939: 87, Engelhardt, 1946: 182, 184; Duckworth, Eichlin, 1977: 52; Duckworth, Eichlin, 1978b: 5, 26, fig. 22, pl. 2; Solomon, Dix, 1979: 16 Heppner, Duckworth, 1981: 26; Friedlander, 1986: 278, 279, 281284; Eichlin, Duckworth, 1988: 52, 56, text-fig. 13e, pl. 2, figs 6, 8 , 10, pl. A, fig. 9; Pühringer, Kallies, 2004: 17; Taft, Schaper, 2014: 58, 60; Pohl et al., 2016: 206; Moreira et al., 2019: 42, 43.

Melittia grandis var. hermosa - Duckworth, Eichlin, 1973a: 13

Trochilium Grande - Duckworth, Eichlin, 1973a: 3

HOST PLANT. Cucurbita foetidissima Kunth (Cucurbitaceae).

DISTRIBUTION. USA: Kansas, California, Arizona, New Mexico, Texas, Oklahoma; Mexico: Durango.

Eichlinia khmer (Le Cerf, 1917), comb.n.

"Melittia Khmer n. sp." — Le Cerf, 1917: 161, pl. 475, fig. 3916. Type locality: "Cambodge, Angkor, ...". Obviously, this type locality is wrong due to mislabeling. It must be located somewhere in North or Central America [Arita, Gorbunov, 1996 b]. Holotype o (MNHP).

Melittia khmer — Dalla Torre, Strand, 1925: 145; Gaede, 1933 791, pl. 95, row f; Heppner, Duckworth, 1981: 27; Arita, Gorbunov, 1996b: 184, figs 39, 40, 58a-d; Pühringer, Kallies, 2004: 17.
Melittia chmer - Hampson, 1919: 93.

HOST PLANT. Unknown.

DISTRIBUTION. North America (?).

Eichlinia magnifica (Beutenmüller, 1899), comb.n.

"Melittia magnifica, sp. nov." — Beutenmüller, 1899: 151. Type locality: "Austin, Texas." [=USA: Texas, Austin]. Holotype + ( AMNH).

Mellitia magnifica — Beutenmüller, 1901: 236, pl. 29, fig. 5; Dyar, 1902: 364; Dalla Torre, Strand, 1925: 146; Zukowsky, 1936: 1250, pl. 179, row c; McDunnough, 1939: 87; Engelhardt, 1946: 182, 191; Duckworth, Eichlin, 1973a: 16; Duckworth, Eichlin, 1977: 52; Solomon, Dix, 1979: 16; Heppner, Duckworth, 1981: 27; Eichlin, Duckworth, 1988: 52, 58, pl. 2, fig. 12; Brown, 2004: 102; Pühringer, Kallies, 2004: 17; Pohl et al., 2016: 206.

HOST PLANT. Cucurbita argyrosperma Huber (Cucurbitaceae).

DISTRIBUTION. USA: Texas; Mexico: Baja Californis.

Eichlinia pulchripes (Walker, 1856), comb.n.

"Melittia pulchripes." - Walker, 1856: 67. Type locality: "Venezuela.". Lectotype + (BMNH), designated by Duckworth, Eichlin 1978: 21.

= "Melittia riograndensis Brèthes, n. sp." — Brèthes, 1920: 284. Type locality: “... Pelotas" [= Brazil: Rio Grande do Sul, Pelotas]. Lectotype $\sigma^{\top}$ (MACN), designated by Becker, Eichlin, 1984: 14.

= Melittia pulchripes dangeloi - Köhler, 1941: 10. Type locality: "Corrientes, ..." [= Argentina: Corrientes]. Lectotype $\sigma^{7}$ (IML), designated by Duckworth, Eichlin 1978a: 21.

= Melittia satyriniformis auct., nec Melittia satyriniformis Hübner, 1831 [“1825”]

Melittia pulchripes - Walker, 1856: 67; Dalla Torre, Strand, 1925: 147; Zukowsky, 1936: 1249, pl. 179, row b; Duckworth, Eichlin, 1978a: 21; Heppner, Duckworth, 1981: 27; Becker, Eichlin, 1984: 14; Pühringer, Kallies, 2004: 15.

Melittia pulchripes d'angeloi — Duckworth, Eichlin, 1978a: 21.

Melittia riograndensis - Zukowsky, 1936: 1253; Becker, Eichlin, 1984: 13, 14

HOST PLANT. Unknown.

DISTRIBUTION. Guatemala; Honduras; Venezuela; Colombia; Brazil: Pará.

Eichlinia snowii (Hy Edwards, 1882), comb.n.

“Melittia Snowii. n. s." — Edwards, 1882: 53. Type locality: "Kansas, ..." [= USA: Kansas]. Lectotype o" (AMNH), designated by Duckworth, Eichlin, 1973a: 27.

Mellitia snowii — Beutenmüller, 1892: 171; Beutenmüller, 1896: 113; Beutenmüller, 1899: 149, 150; Beutenmüller, 1901: 234, pl. 29, fig. 2; Dalla Torre, Strand, 1925: 148; Zukowsky, 1936: 1249, pl. 179, row b; Engelhardt, 1946: 182, 186; Duckworth, Eichlin, 1973a: 27; Duckworth, Eichlin, 1977: 52; Solomon, Dix, 1979: 16; Heppner, Duckworth, 1981: 27; Friedlander, 1986: 279, 281-283; Eichlin, Duckworth, 1988: 52, 54, text-fig. 13f, pl. 2, fig. 7; Eichlin, 1995: 48; Pühringer, Kallies, 2004: 17; Taft, Schaper, 2014: 60; Pohl et al., 2016: 206; Moreira et al., 2019: 42, 43.

Melittia snowi - McDunnough, 1939: 87; Stallings, Turner, 1944: 31

HOST PLANT. Cucurbita foetidissima Kunth (Cucurbitaceae).

DISTRIBUTION. USA: Colorado, Nebraska, Kansas, Arizona, New Mexico, Oklahoma, Texas.

Acknowledgements. I would like to express my sincere gratitude to Dr. Gilson R.P. Moreira (Universidade Federal do Grande do Sul, Porto Alegre, Brazil) for sending to me papers of some South American authors. I am indebted to Mr. Vlad Proklov (London, England) for carefully checking the English of an advanced draft. The study was conducted using the equipment of the Joint Usage Center "Instrumental meth- 
ods in ecology" at the A.N. Severtsov Institute of Ecology and Evolution, Russian Academy of Sciences (Moscow, Russia).

\section{References}

Arita Y., Gorbunov O.G. 1995. A revision of the Melittia types (Lepidoptera, Sesiidae) kept in the Hope Entomological Collection, Oxford University, UK // Trans. lepid. Soc. Japan. Vol.46. No.4. P.185-205.

Arita Y., Gorbunov O.G. 1996a. New and unrecorded clearwing moths of the genus Melittia Hübner, [1819] (Lepidoptera, Sesiidae) from Thailand // Trans. lepid. Soc. Japan. Vol.47. No.3. P.157-173.

Arita Y., Gorbunov O.G. 1996b. A revision of Ferdinand Le Cerf's clearwing moth types (Lepidoptera, Sesiidae), kept at the Paris Museum // Jpn. J. syst. Ent. Vol.2. No.2. P.137-187.

Barnes W., Benjamin F.H. 1925. Change of a preoccupied name (Lepidoptera: Aegeriidae)// Proc. Ent. Soc. Washington. Vol.27. No.1. P.14.

Barnes W., Lindsey A.W. 1922. Descriptions of two new species of Aegeriidae (Lep.) // Bull. Brooklyn Ent. Soc. (N.S.). Vol.18. No.4. P.122-123.

Bartel M. 1912. 24. Familie: Aegeriidae (Sesiidae) // Seitz A (Hrsg.). Die Gros-Schmetterlinge der Erde. Bd.2. Die Palaearktischen Spinner \& Schwärmer. Stuttgart: A Kernen Verlag. S.375-416. P1.51-52.

Bartsch D. 2016. Melittia fiebigi spec. nov. and Afromelittia caerulea spec. nov., two new Melittiini from southern Africa (Lepidoptera: Sesiidae) // Annals Ditsong Nation. Mus. Nat. Hist. Vol.6. No.7. P.109-115.

Becker V.O., Eichlin T.D. 1984. Correct name for the Neotropical squash-vine borer (Sesiidae: Melittia) // J. Lepidopterists' Soc. Vol.38.No.1. P.13-14.

Beutenmüller W. 1892. List of types of Lepidoptera in the Edwards collection of Insects // Bull. Amer. Mus. Nat. Hist. Vol.4. P.167198.

Beutenmüller W. 1896. Critical review of the Sesiidae found in America, north of Mexico // Bull. Amer. Mus. Nat. Hist. Vol.8. P.111-148.

Beutenmüller W. 1899. Synopsis of the species of Melittia of America, North of Mexico, with description of a new species // Bull. Amer. Mus. Nat. Hist. Vol.12, 149-151.

Beutenmüller W. 1901. Monograph of the Sesiidae of America, North of Mexico // Memoirs Amer. Mus. Nat. Hist. Vol.1. No.6. P.217-352. Pls.29-36.

Brown J.W. 2004. Preliminary assessment of Lepidoptera diversity on the peninsula of Baja California, Mexico, with a list of documented species // Folia Ent. Mexicana (N.S.). Vol.42. No.1. P.87-114.

Brown L.N., Mizel R.F.III 1993. The clearwing borers of Florida (Lepidoptera: Sesiidae) // Tropical Lepid. Vol.4. Suppl.4. P.1-21.

Brèthes J. 1920. Insectos útiles y dañinos de Rio Grande do Sul y de La Plata // Anales Soc. Rural Argentina. Vol.54. P.281-290, 307-308.

Canhilal R., Carner G.R., Griffin R.P., Jackson D.M., Alverson D.R. 2006. Life history of the squash vine borer, Melittia cucurbitae (Harris) (Lepidoptera: Sesiidae) in South Carolina // J. Agric. Urban Entomol. Vol.23. No.1. P.1-6.

Capinera J.L., 2008. Squash Vine Borer, Melittia cucurbitae (Harris) (Lepidoptera: Sesiidaae) // Capinera J.L. (ed.). Encyclopedia of entomology. Dordrecht: Springer. P.3533-3536.

Dalla Torre K.W., Strand E. 1925. Aegeriidae // Strand E. (Hrsg.) Lepidopterorum Catalogus. Bd.31. Berlin: W. Junk. 202 S.

Druce H. 1883. Insecta. Lepidoptera-Heterocera. Vol. I // Godman D., Salvin O. (eds.). Biologia Centrali-Americana; or contributions to the knowledge of the fauna and flora of Mexico and Central America. Vol.39. London: Taylor and Francis. P.1-32, P1.5.

Druce H. 1892. Description of a new genus and some new species of Heterocera from Central America // Annals Mag. Nat. Hist. Ser.6. Vol.9. P.275-279.
Druce H. 1896. Insecta. Lepidoptera-Heterocera. Vol. II // Godman D., Salvin O. (eds.). Biologia Centrali-Americana; or contributions to the knowledge of the fauna and flora of Mexico and Central America. Vol.40. London: Taylor and Francis. P.273336.

Druce H. 1897. Insecta. Lepidoptera-Heterocera. Vol. III // Godman D., Salvin O. (eds.). Biologia Centrali-Americana; or contributions to the knowledge of the fauna and flora of Mexico and Central America. Vol.41. London: Taylor and Francis. P.69-72.

Duckworth W.D., Eichlin T.D. 1973a. The type-material of North American clearwing moths (Lepidoptera: Sesiidae) // Smithsonian Contr. Zool. No.148. P.1-34.

Duckworth W.D., Eichlin T.D. 1973b. New species of clearwing moths (Lepidoptera: Sesiidae) from North America // Proc. Entom. Soc. Washington. Vol.75. No.2. P.150-159.

Duckworth W.D., Eichlin T.D. 1977. A classification of the Sesiidae of America north of Mexico (Lepidoptera: Sesioidea) // Occasional papers in Entomology. Vol.26. P.1-54.

Duckworth W.D., Eichlin T.D. 1978a. The type-material of Central and South American clearwing moths (Lepidoptera: Sesiidae) // Smithsonian Contr. Zool. No.261. P.1-28.

Duckworth W.D., Eichlin T.D. 1978b. The clearwing moths of California (Lepidoptera: Sesiidae) // Occasional papers in Entomology. Vol.27. P.1-90.

Dyar H.G. (1902) A list of North American Lepidoptera and key to the literature of this order of Insects // Bull. U. S. Nat. Mus. Vol.52. $x i x+723$ pp.

Edwards H. 1880. Descriptions of some new forms of Aegeriidae // Bull. Brooklyn Entom. Soc. Vol.3. No.8. P.71-72.

Edwards H. 1882. Notes on N. American Aegeridae, with descriptions of new forms // Papilio. Vol.2. No.4. P.52-57.

Eichlin T.D. 1975. Clearwing moth borers of cucurbits // Natl. Pest Control Operators News. Vol.35. P.4-7.

Eichlin T.D. 1992. Clerawing moths of Baja Californis, Mexico (Lepidoptera: Sesiidae) // Trop. Lepid. Vol.3. No.2. P.135-150.

Eichlin T.D. 1995. New data and a redescription for Melittia oedipus, an African vine borer (Lepidoptera, Sesiidae) // Trop. Lepid. Vol.6. No.1. P.47-51.

Eichlin T.D., Duckworth W.D. 1988. Sesioidea: Sesiidae // R.B. Domonick et al. The Moths of America North of Mexico. Fasc.5.1. Washington, D.C.: the Wedge Entomological research foundation. 76 pp., 6 pls.

Engelhardt G.P. 1924. Field notes on Western clear wing moths (Aegeriidae) // Bull. Brooklyn Entom. Soc. (N.S.). Vol.19. No.4. P.125-127.

Engelhardt G.P. 1946. The North American clear-wing moths of the family Aegeriidae // Bull. United States Nat. Mus. Vol.190. iv $+222 \mathrm{pp}$.

Fletcher D.S., Nye I.W.B. 1982. Bombycoidea, Castnoidea, Cossoidea, Mimallonoidea, Sesioidea, Sphingoidea, Zygaenoidea // Nye I.W.B. (ed.). The generic names of oths of the world. Vol.4. London: Trustees of the British Museum (Natural History). XIV $+192 \mathrm{pp}$.

Friedlander T.P. 1986. A new squash borer from Mexico (Lepidoptera: Sesiidae) // J. Research Lepid. Vol.24. No.4. P.277-288.

Gaede M. 1933. 23. Familie: Aegeriidae // Seitz A. (Hrsg.). 19261930. Die Gross-Schmetterlinge der Erde. Bd.10. Die indoaustralischen Spinner und SchwÃormer. Stuttgart: A. Kernen Verlag. S.775-802. P1.94-95.

Gorbunov O.G. 2014. A new species of the genus Melittia Hübner, 1819 ["1816"] from the island of Lombok, Indonesia // Far Eastern Entomologist. No.284. P.13-18.

Gorbunov O.G. 2015. Contributions to the study of the Ethiopian Lepidoptera. I. The genus Melittia Hübner, 1819 ["1816"] with description of a new species // Zootaxa. Vol.4033. No.4. P.543554.

Gorbunov O.G. 2017. On the taxonomy and morphology of Leuthneria ruficincta (Lepidoptera, Sesiidae) // Zootaxa. Vol.4244. No.1. P.127-136.

Gorbunov O.G. 2020. To the morphology and synonymy of insufficiently known Melittia rutilipes Walker, 1865 ["1864"] (Lepidoptera: Sesiidae) // Russian Entomol. J. Vol.29. No.1. P.87-92. 
Gorbunov O.G., Arita Y. 1995. New taxa of the tribe Melittiini (Lepidoptera, Sesiidae) from the Oriental Region // Tinea. Vol.14. No.3. P.149-156.

Gorbunov. O.G., Arita Y. 1996. New and little-known Oriental Melittia Hübner (Lepidoptera, Sesiidae), from the collection of Muséum d'histoire naturelle, Genève // Revue suisse Zool. Vol.103. No.2. P.323-338.

Gorbunov O.G., Arita Y. 1997. A revision of Ferdinand Le Cerf's clearwing moth types (Lepidoptera, Sesiidae), kept at the Paris Museum. II. Melittiini in the Afrotropical Region // Jpn. J. syst. Ent. Vol.3. No.2. P.289-323.

Hampson G.F. 1892. Family Sesiidae // Hampson G.F. The Fauna of British India, including Ceylon and Burma (Moths). Vol.I. London: Taylor and Francis; Calcutta: Thacker, Spink, \& Co.; Bombay: Thacker \& Co., Berlin: R Friedländer \& sohn. P.187206.

Hampson G.F. 1919. A classification of the Aegeriadae [sic] of the Oriental and Ethiopian Regions // Novitates Zoologicae. Vol.26. No.1. P.46-119.

Harris T.W. 1828. Insects // New England Farmer. Vol.7. No.5 P.33-34.

Heppner J.B., Duckworth W.D. 1981. Classification of the Superfamily Sesioidea (Lepidoptera, Ditrysia) // Smithsonian Contr. Zool. No.314. P.i-iii, 1-144.

Howe W.L. 1950. Biology and host relationships of the Squash Vine Borer // J. Econom. Ent. Vol.43. No.4. P.480-483.

Howe W.L., Rhodes A.M. 1973. Host relationships of the Squash Vine Borer, Melittia cucurbitae with species of Cucurbita // Ann. Ent. Soc. America. Vol.66. No.2. P.266-269.

Hübner J. 1816-[1826]. Verzeichniß bekannter Schmettlinge [sic]. Augsburg. $432 \mathrm{~S}$.

Hübner J. 1831 [“1825”]. Zuträge zur Sammlung exotischer Schmetterlinge, bestehend in Bekanntmachung einzelner Geschlechter neuer oder seltener, nichteuropäischer Gattungen. Dritter Hundert. Augsburg. $40+$ [9] S. Fig.401-600.

ICZN (The International commission on zoological nomenclature) 1999. International Code of zoological nomenclature. 4th Ed London: The International trust for zoological nomenclature, xxix +309 pp.

Jackson D.M., Canhilal R., Carner G. 2005. Trap monitoring squash vine borers in cucurbits // J. Agric. Urban Entomol. Vol.22. No.1. P.27-39.

Kallies A. 2020. The clearwing moths (Lepidoptera, Sesiidae) of Australia, New Guinea and the Pacific Islands // Zootaxa Vol.4833. No.1. P.1-64.

Kallies A., Arita Y., Min W. 2016. A new species and new records of Melittiini from China and Vietnam (Lepidoptera, Sesiidae) // Zootaxa. Vol.4205. No.2. P.162-170.

Klun J.A., Schwarz M., Leonhardt B.A., Cantelo W.W. 1990. Sex pheromone of the female squash vine borer (Lepidoptera: Sesiidae) // J. Entomol. Science. Vol.25. No.1. P.64-72.

Köhler P. 1941. Aegeriidae argentinas del Instituto Miguel Lillo (Lep. Het.). Buenos Aires: "Coni". P.3-12.

Krinski D. 2015. First report of Squash Vine Borer, Melittia cucurbitae (Harris, 1828) (Lepidoptera, Sessidae [sic!]) in Brazil and South America: distribution extension and geographic distribution map // CheckList. Vol.11. No.3. P.1-3.

Le Cerf F. 1916. Explication des planches // Études Lépid. Comparée. Vol.12. No.1. P.7-14. Pls373-381.

Le Cerf F. 1917. Contributions à l'étude des Aegeriidae. Description et Iconographie d'Espèces et de Formes nouvelles ou peu connues // Études Lépid. Comparée. Vol.14. P.137-388. Pls.475-481.

McDunnough J. 1939. Check list of the Lepidoptera of Canada and the United States of America. Part II. Microlepidoptera // Mem. Southern California Acad. Sci. Vol.2. No.1. P.1-171.

Middleton E. 2018. Biology and management of Squash Vine Borer (Lepidoptera: Sesiidae) // J. Integrated Pest Management. Vol.9.No.1. P.1-8

Moreira G.R.P., Gorbunov O.G., Fochezato J., Gonçalves G.L. 2019. A peculiar new species of gall-inducing, clearwing moth (Lep- idoptera, Sesiidae) associated with Cayaponia in the Atlantic Forest // ZooKeys. Vol.866. P.39-63.

Pohl G.R., Patterson B., Pelham J.P. 2016. Annotated taxonomic checklist of the Lepidoptera of North America, North of Mexico. Working paper published online by the authors at ResearchGate. net. $766 \mathrm{pp}$.

Powell J.A. 2005. Assessment of inventory effort for Lepidoptera (Insecta) and the status of endemic species on Santa Barbara Island California // Garcelon D.K., Schwemm C.A. (eds.). Proceedings of the Sixth California Island Symposium. Santa Barbara, CA: Santa Barbara Museum of Natural History. P.351371.

Pühringer F., Kallies A. 2004. Provisional check list of the Sesiidae of the world (Lepidoptera: Ditrysia) // Mitt. Ent. Arb.gem. Salzkammergut. Bd.4. S.1-85.

Pühringer F., Kallies A. 2017. Checklist of the Sesiidae of the world (Lepidoptera: Ditrysia). Available at http://www.sesiidae.net/ Checklst.htm (Accessed on 26 April, 2020).

San Martín-Romero E., Martínez-Rosas R., Espinosa-Mendoza M., Núnñez-Camargo C., Landa-Cadena G., Salinas-Castro A. 2019. Primer reporte de Melittia calabaza (Duckworth y Eichlin) (Lepidoptera: Sesiidae) en el Estado de Veracruz, México // Revista Chilena Ent. Vol.45. No.4. P.681-687.

Snow F.H. 1905. Some Results of the University of Kansas Entomological Expeditions to Arizona in 1904 and 1905 // Trans. Kansas Acad. Sci. Vol.20. P.155-181.

Solomon J.D., Dix M.E. 1979. Selected bibliography of the clearwing borers (Sesiidae) of the United States and Canada // U.S. Dept. Agric. Forest Serv. Gen. Tech. Rep. No.SO-22. 18 pp.

Sondak K.E. 1981. The Southwestern squash vine borer, Melittia calabasa. A thesis submitted to the Faculty of the Department of Entomology in Partial Fulfillment of the requirements for the degree of Master of Sciences. University of Arizona. Ix +63 pp., 7 figs.

Špatenka K., Gorbunov O., Laštůvka Z., Toševski I., Arita Y. 1999. Sesiidae, Clearwing Moths // Naumann C.M. (ed.). Handbook of Palaearctic Macrolepidoptera. Vol.1. Wallingford: Gem Publishing Company. 569 pp., 57 pls.

Stallings D.B., Turner J.R. 1944. Notes on Lepidoptera // J. Kansas Ent. Soc. Vol.17. No.1. P. 29-31.

Stoll C. 1782. De Uitlandsche Kapellen voorkomende in de drie waereld-deelen Asia, Africa en America. Deel iv. Amsteldam: S.J. Baalde, pp. 165-252, pls ccclxxi-cccc.

Strecker F.H.H. 1881. Description of a new species of Trochilium // Canadian Entomologist. Vol.13. No.7. P.156

Taft W.H. jr., Schaper N. 2014. Uncommon clearwing moths (Sesiidae) from southeast Arisona // News Lep. Soc. Vol.56. No.2. P.58-61.

The Plant List. 2010. Version 1. Published on the Internet. http:// www.theplantlist.org/ (accessed 18 August 2020).

Thompson B.G. 1929. Moths of the family Aegeriidae occurring in Oregon, with notes // Pan-Pacific Entomol. Vol.5. No.1. P.117122.

Walker F. 1856. List of the specimens of lepidopterous Insects in the collection of the British Museum. Part VIII. Sphingidae. London: British Museum. 271 pp.

Walsingham M.A. 1913. Biologia Centrali-Americana. Insecta. Lepidoptera-Heterocera. Vol. IV. Tineina, Pterophorina, Orneodina, and Pyralidina and Hepialina (part.). London: Taylor and Francis. P.169-224, P1.6.

Westwood J.O. 1848. The Cabinet of Oriental Entomology; being a selection of some of the rarer and more beautiful species of Insects, natives of India and the adjacent islands, the greater portion of which are now for the first time described and figured. London: William Smith. 88 pp. 42 pls.

Zukowsky B. 1936-1937. Familie: Aegeriidae // Seitz A. (Hrsg.), 1907-1913. Die Gros-Schmetterlinge der Erde. Bd.6. Die Amerikanischen Spinner und Schwärmer. Stuttgart: A Kernen Verlag. S.1215-1262, Taf.176-180, 185. 